La taylorisation des traitements sur le profil moléculaire Un essai original signé par une équipe du MD Anderson : la «taylorisation » du traitement basé sur le profil moléculaire des tumeurs. À chaque anomalie détectée, un traitement ciblé était proposé. Cette phase I proposait de traiter les patients dont la tumeur exprimait une anomalie moléculaire par le traitement ciblé disponible qui correspondait ou par un traitement «standard».

Résultats probants en taux de réponse, PFS et survie ! (Abstract CRA 2500 - A.M. Tsimberidou et al.)

Crizotinib dans les CBNPC ALK+: la suite (cf. Oncomagazine $\mathrm{n}^{\circ}$ 3-2010)

À partir des résultats d'une phase I. 119 patients dont 116 évaluables. $44 \%$ avaient reçu $\geq$ 3 lignes de traitement antérieur. Réponse objective de $61 \%$, dont 2RC et 69 RP. Avec un délai jusqu'à la réponse de 8 semaines, une durée médiane de réponse de 48 semaines et une SSP médiane de 10 mois, ces résultats confirment la robustesse des premières données communiquées à l'ASCO 2010. La tolérance est marquée par des effets secondaires majoritairement de grades $1 / 2$ à type de troubles visuels et digestifs qui apparaissent rapidement mais régressent généralement dès l'arrêt du traitement.

À vos tests, prêts, partez! (Abstract 2501 - Ross Camidge et al.)

\section{ASCO 2011 : Ce qui ne vous aura pas échappé}

La prévention du cancer du sein par l'exemestane chez les femmes ménopausées à risque de développer un cancer du sein réduit le risque de survenue du cancer de $65 \%$ : conclusions de l'essai du $\mathrm{NCl}$ canadien MAP.3 publié le jour même dans le NEJM en ligne (NEJM.org) et sur toutes les ondes radiophoniques et télévisées.

Cet essai versus placebo a recruté 4560 femmes ménopausées de plus de 60 ans, ayant au moins un facteur de risque. Suivi médian de 35 mois. 11 vs 32 cancers invasifs détectés (exemestane vs placebo), réduction relative du risque de $65 \%(p=0,002)$.

Incidence annuelle de cancer invasif ou non : $0,35 \%$ vs $0,77 \%$ $(\mathrm{HR}=0,47 ; p=0,004)$.

Au prix d'un impact modéré sur la qualité de vie, l'exemestane réduit significativement la survenue d'un cancer chez les femmes ménopausées à risque accru de cancer du sein. (LBA504 - P.Goss et al.)

Du nouveau dans les mélanomes!

- Le vemurafenib, inhibiteur de BRAF muté $(50 \%$ des mélanomes), prolonge la survie globale. Phase III dacarbazine vs vemurafenib sur 675 patients avec un mélanome non prétraité de stades IIIC ou IV mutés V600E sur BRAF.

À 3 mois : réduction du risque de décès de $63 \%(p<0,0001)$, réduction du risque de rechute de $74 \%, p<0,0001)$, avantage en réponse objective : $48,4 \%$ vs $5,5 \%$.

Effets secondaires : rashs cutanés, photosensibilité, élévation des enzymes hépatiques, arthralgies. Le vemurafenib un candidat pour un nouveau standard dans les mélanomes mutés BRAF ? (Abstract LBA4 - P.B. Chapman, A. Hauschild, C. Robert et al. Improved Survival with Vemurafenib in Melanoma with BRAF V600E Mutation. N Engl J Med 2011;364:2507-16.)

- L'ipilimumab, inhibiteur CTLA4, améliore la survie globale. Phase III dacarbazine \pm ipilumumab chez 502 patients non prétraités. Survie à 1 an :47,3\% vs 36,3\%; à 2 ans : $28,5 \%$ vs $17,9 \%$; à 3 ans : $20,8 \%$ vs $12,2 \%$. Médianes de survie globales respectivement de 11,2 mois et 9,1 mois ; bénéfice en survie retrouvé dans l'ensemble des sous-groupes.

Avec une réduction du risque de progression de $24 \%$, à 12 semaines, les médianes de SSP sont très voisines ( 2,8 vs 2,6 mois). Lié au mécanisme d'action d'un immunomodulateur d'action retardée par rapport à une chimiothérapie classique ? Le profil de tolérance est favorable. $1^{\text {re }}$ étude à démontrer un bénéfice en survie durable avec chimiothérapie + immunothérapie. D'autres combinaisons à tenter? (Abstract LBA5 - J.D. Wolchok, C. Robert, L. Thomas, I. Bondarenko et al. Ipilimumab plus Dacarbazine for Previously Untreated Metastatic Melanoma. N Engl J Med 2011;364:2517-26.) •

Au moment où nous mettons sous presse ce numéro, nous apprenons le décès de David Servan-Schreiber. Au-delà des controverses sur alimentation et cancer, sachons reconnaître un homme de conviction qui a su donner un bel exemple de dignité. Que ses enfants, son épouse et toute sa famille et amis trouvent ici le témoignage de sympathie de l'équipe d'Oncomagazine. Puissions nous tous, à son image, rester lucide et digne aux moments ultimes de notre vie.

Gain de survie dans les GIST : imatinib 1 an vs 3 ans en adjuvant

Cette étude de phase III a comparé un traitement sur 1 an vs un traitement de 3 ans par imatinib chez 400 patients à haut risque de récidive.

À 5 ans : SSP : $65,6 \%$ vs $47,9 \% ; \mathrm{HR}: 0,46, p<0,0001$; SG : $92 \%$ vs $81,7 \%$, HR : 0,45, $p<0,019$.

Peu de résistance au traitement dans l'ensemble bien toléré avec principalement une anémie, fatigue, diarrhée et crampes musculaires.

Arrêt du traitement par effets secondaires : 7,7 \% (1 an) et 13,7 \% (3 ans). (Abstract LBA 1 H. Joensuu et al.)

La maintenance dans les CB non épidermoïdes : ça marche!

Cet essai de phase III comparait une maintenance par pemetrexed jusqu'à progression à un placebo chez des patients avec un CB non épidermoïdes avancé préalablement traités par pemetrexed + cisplatine en traitement initial non progressifs après 4 cycles.

Le risque de récidive a été réduit de $38 \%$.SSP médianes : 4,1 mois vs 2,8 mois, $p=$ 0,00006.

Contrôle de la maladie : 71,8 \% vs $59,6 \%, p=0,009$.

Le profil de tolérance est favorable et conforme à celle connue du pemetrexed en monothérapie. Un nouveau standard serait-il en chemin? (CRA 7510 - L.G. Paz-Ares et al.)

Toutes les références ASCO sont issues de : J Clin Oncol 2011 ;29, Issue 18, Suppl June 20

Anne Ponzio-Prion, IGR, Villejuif 